\title{
Mass Cytometry Reveals the Immaturity of Circulating Neutrophils during SIV Infection
}

\author{
Julien Lemaitre ${ }^{\mathrm{a}}$ Antonio Cosma ${ }^{\mathrm{a}}$ Delphine Desjardins ${ }^{\mathrm{a}}$ Olivier Lambotte ${ }^{\mathrm{a}, \mathrm{b}}$ \\ Roger Le Grand ${ }^{\text {a }}$ \\ aINSERM U1184, Immunology of Viral Infections and Autoimmune Diseases, IDMIT Department, IBFJ, CEA -

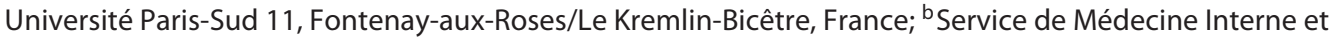 \\ Immunologie Clinique, Hôpital Bicêtre, Assistance Publique - Hôpitaux de Paris, Le Kremlin-Bicêtre, France
}

\section{Keywords}

AIDS - Antiretroviral treatment - Mass cytometry ·

Neutrophils $\cdot$ Nonhuman primate $\cdot$ Phagocytosis

\section{Abstract}

The infected host fails to eradicate HIV-1, despite significant control of viral replication by combinational antiretroviral therapy. Here, we assessed the impact of HIV infection on immune-cell compartments in a SIVmac251 nonhuman primate infection model, which allowed the choice of contamination route, time of infection, and treatment follow-up. We performed high-throughput multiparameter single-cell phenotyping by mass cytometry to obtain a global vision of the immune system in blood and bone marrow. Circulating polymorphonuclear neutrophils (PMNs) with impaired phagocytosis had altered surface expression of CD62L and CD11b during early chronic infection. The initiation of combinational antiretroviral treatment during primary infection did not restore PMN function. The maturation state of PMNs was highly altered during late chronic SIV infection, showing a primarily immature phenotype. Our results provide new insights into PMN involvement in the pathogenesis of HIV infection and may play a role in the establishment and maintenance of chronic immune activation.

(C) 2019 The Author(s)

Published by S. Karger AG, Basel

\section{Introduction}

Combinational antiretroviral treatment (cART) has increased life expectancy and the quality of life of HIV-infected patients. Nevertheless, lifelong treatment is necessary to control virus replication because of the inability of cART and host defenses to eradicate the virus. HIV persistence is associated with chronic inflammation in many infected patients, leading to an increased risk of comorbidities, such as cardiovascular diseases [1], neurocognitive disease [2], kidney disease [3], and cancer [3]. The proinflammatory cytokine IL-6 and soluble (s)CD14 and sCD163 levels are associated with an increased risk of mortality for both treated and untreated HIV-infected patients [4-6]. Inflammatory markers can also be directly linked to the monocyte and

\section{KARGER}

E-Mail karger@karger.com www.karger.com/jin

\section{() 2019 The Author(s) \\ Published by S. Karger AG, Basel \\ Karger \\ Open access}

This article is licensed under the Creative Commons AttributionNonCommercial-NoDerivatives 4.0 International License (CC BYNC-ND) (http://www.karger.com/Services/OpenAccessLicense). Usage and distribution for commercial purposes as well as any distribution of modified material requires written permission.
Prof. Roger Le Grand

Immunology of Viral Infections and Autoimmune Diseases, CEA -

Université Paris-Sud 11 - INSERM U1184

18, route du Panorama

FR-92265 Fontenay-aux-Roses (France)

E-Mail roger.le-grand@cea.fr 
macrophage activation associated with microbial translocation that occurs in HIV infection [7]. Finally, chronic inflammation contributes to exhaustion of the adaptive immune system and T-cell activation. Polymorphonuclear neutrophils (PMNs) are myeloid cells that are also associated with chronic inflammation in autoimmune diseases, such as rheumatoid arthritis [8]. However, few studies have been conducted on their role in HIV infection. In chronic HIV-1 or SIV infection, neutrophils show an activated profile [9], increased sensitivity to apoptosis [10], and impaired phagocytosis $[11,12]$. Neutrophils have been shown to suppress interferon $-\gamma$ production by CD8+ T cells in chronic HIV-1 infection via the PD-L1/PD-1 axis and were more prone to degranulation, contributing to chronic immune activation in patients [13].

It is currently difficult to obtain a global view of the impact of HIV infection on the immune system, especially on myeloid cells. Available studies have several limitations. Bone marrow, which is the differentiation and maturation compartment of myeloid cells, is still poorly characterized in HIV infection. In addition, innate immunity plays a major role in the first steps of any infection, but the study of the first days of infection is rare in humans because of limited sample availability. Cynomolgus macaques infected with SIVmac251 are a suitable model to overcome such limitations, because many aspects of this model parallel those of HIV-1 physiopathology [14-17] and the hematopoietic system of macaques is similar to that of humans $[18,19]$.

We characterized myeloid-cell heterogeneity in cynomolgus macaque blood and bone marrow using recently developed mass cytometry technology, based on metal isotope-conjugated antibodies, that enables the detection of more than 40 markers per cell [20], providing major advantages over conventional flow cytometry. The background is very low because heavy metals are not present in biological specimens and there is no need for channel compensation, as required for laser-based flow cytometry. Our analysis suggests significant modification of the myeloidcell compartment, especially circulating PMNs. During the early chronic phase, PMNs had surface expression of PMN CD62L decreased and that for CD11b increased, suggesting that PMNs were primed in SIV infection. In parallel, circulating PMNs demonstrate a mature phenotype but phagocytosis was impaired. In late chronic SIV infection, blood PMNs consisted of mostly immature neutrophils. These results highlight the need for further study of tissue myeloid-cell dynamics in an HIV infection model to better understand chronic inflammation and design new treatments for HIV remission and a functional cure.

\section{Materials and Methods}

Nonhuman Primates and Whole-Blood Collection

Twenty-nine cynomolgus macaques (Macaca fascicularis), imported from Mauritius, were housed at the IDMIT infrastructure animal facility at the CEA, Fontenay-aux-Roses, France. Animals expressing the H6 MHC haplotype were excluded because of their increased natural capacity to control SIVmac251 infection [21]. Seventeen animals were infected by intravenous inoculation with 1,000 animal infectious dose 50\% (AID50) of pathogenic SIVmac251 isolate and followed for 18 months [22]. Six SIV-infected animals were subcutaneously treated daily, starting from 28 days after infection, with a combination of two nucleoside reverse-transcriptase inhibitors, emtricitabine ( $40 \mathrm{mg} / \mathrm{kg}$ ) and tenofovir disoproxil fumarate $(5.1$ $\mathrm{mg} / \mathrm{kg})$, and one integrase inhibitor, dolutegravir $(2.5 \mathrm{mg} / \mathrm{kg})$ [23]. Twelve healthy macaques were used as an uninfected control group.

Three healthy animals and 2 SIV-infected animals, which were sampled 18 months after inoculation, were used for mass cytometry analysis. In addition, 15 animals in the early chronic phase of SIV infection (120-288 days after inoculation) were used, along with 9 uninfected macaques (online suppl. Table 1; for all online suppl. material, see www.karger.com/doi/10.1159/000499841), for flow cytometry analysis. Blood was drawn, and bone marrow aspirates were collected under anesthesia, consisting of an intramuscular injection of $10 \mathrm{mg} / \mathrm{kg}$ ketamine (Rhone-Mérieux, Lyon, France). Blood was collected into lithium-heparin tubes (Vacutainer BD, USA) and bone marrow aspirates from the iliac crest using a $10-\mathrm{mL}$ $18-\mathrm{G}$ syringe containing citrate dextrose $(10 \mathrm{mg} / \mathrm{mL})$.

The macaque study was approved by the Ministère del'Education Nationale, de l'Enseignement Supérieur et de la Recherche (France) and the ethics committee Comité d'éthique en expérimentation animale No. 44 under reference 2015102713323361.02 (APAFIS\#2453). Animals were handled in accordance with national regulations (CEA accreditation No. D92-032-02) and European Directive $(2010 / 63$, recommendation No. 9) in compliance with the Standards for Human Care of the Office for Laboratory Animal Welfare (OLAW, USA) under OLAW Assurance No. A5826-01.

Reagents and Cell Preparation and Storage for Mass Cytometry Blood and bone marrow samples were processed using a previously described procedure, consisting of fixation, red blood cell lysis, and freezing [19], that allows the recovery of all blood leukocyte populations, especially PMNs, which are highly labile and cryosensitive. Briefly, $1 \mathrm{~mL}$ of whole blood or bone marrow aspirate was mixed with $10 \mathrm{~mL}$ fixation mixture and incubated on ice for $10 \mathrm{~min}$. After centrifugation, red blood cells were lysed by adding $10 \mathrm{~mL}$ Milli-Q water at room temperature (RT) and incubating the samples for 20 min. After two washes with $1 \times$ modified Dulbecco's phosphate-buffered saline (DPBS), the cells were counted and stored at $-80^{\circ} \mathrm{C}$ in fixation mixture at a final concentration of $15 \times 10^{6}$ fixed leukocytes/ $\mathrm{mL}$ and distributed into aliquots containing $3 \times 10^{6}$ cells.

\section{Staining and CyTOF Acquisition}

Three million fixed cryopreserved cells were thawed at $37^{\circ} \mathrm{C}$, washed twice with PBS/0.5\% bovine serum albumin and incubated on ice for $30 \mathrm{~min}$ with the metal-labeled surface antibodies listed in online supplementary Table 2 . All antibodies were previously tested for cross-reactivity against cynomolgus cells [19]. After two washes with $1 \times$ modified DPBS, the cells were fixed with PBS $/ 1.6 \%$ paraformaldehyde (PFA) at RT for 20 min and permeabilized with 
Fig. 1. Mass cytometry data analysis pipeline. Data were first normalized using internal metal beads to correct instrument variation during the time of acquisition. Cell events were selected within singlets and normalization beads excluded using iridium-191 nucleus staining. Then, cell events were manually gated on the CD66abce/CD3 bivariate plot to exclude CD3/CD66abce double-positive cells, eosinophils that bind all metal complexes on the antibodies. Cell clustering was performed using the SPADE algorithm, grouping phenotypically related cells in a homogenous cluster. The clusters were annotated, and abundance variation was analyzed using the SPADRVizR R-package.

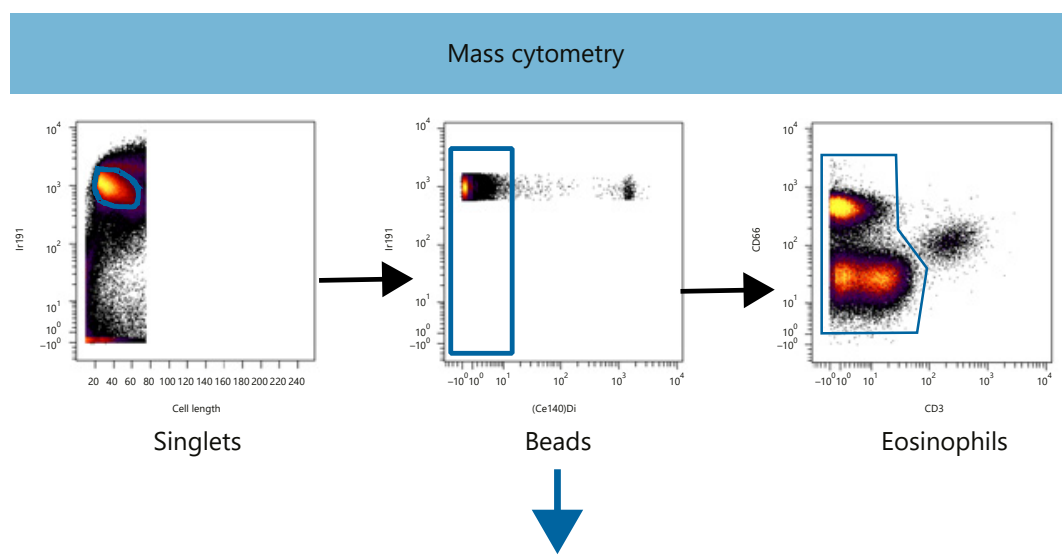

Cell clustering
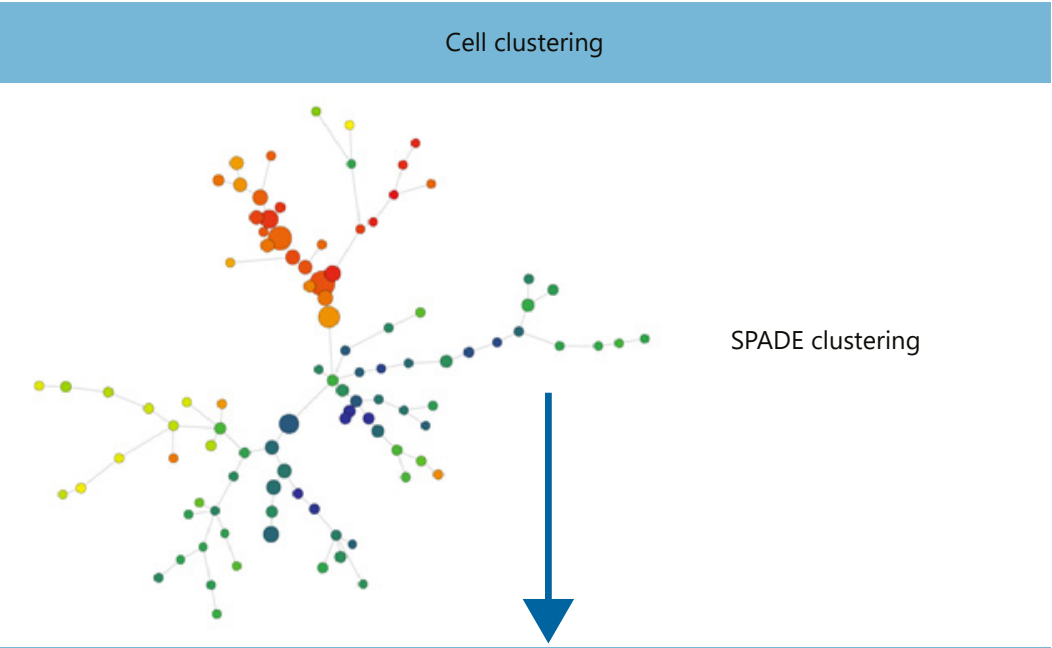

SPADE clustering
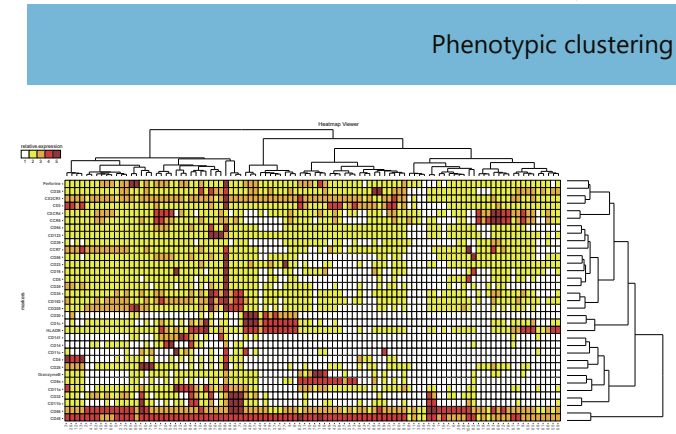

Abundance analysis
$1 \times$ Perm/Wash Buffer (BD Biosciences) at RT for $10 \mathrm{~min}$. Intracellular antibodies and iridium nucleic acid intercalator were incubated on ice for $30 \mathrm{~min}$. After two washes with $1 \times$ modified DPBS, the cells were fixed with PBS/1.6\% PFA at RT for 20 min, centrifuged, and stored overnight in $0.1 \mu \mathrm{M}$ iridium nucleic acid intercalator in PBS/1.6\% PFA. The following day, the cells were washed with Milli-Q water, resuspended in $1 \mathrm{~mL}$ Milli-Q water, and filtered through a $35-\mu \mathrm{m}$ nylon mesh cell strainer (BD Biosciences), before the addition of EQTM Four-Element Calibration Beads (Fluidigm, San Francisco, CA, USA), according to the manufacturer's instructions. Each sample was split in half and run the same day to reduce instrument variation. The number of acquired cells is given in online supplementary Table 3 for each sample.

\section{Data Processing and Analysis for CyTOF}

Data were normalized using Rachel Finck's MATLAB normalizer [24]. Replicates were concatenated using the FCS file concatenation tool (Cytobank, Mountain View, CA, USA). Four-Element Calibration Beads were excluded by manual gating on the $\mathrm{Ce}^{140}$ channel. Singlets were selected on an ${ }^{191}$ Ir DNA intercalator/cell length bivariate plot (Fig. 1). We consistently observed a clear population of cells showing a strong background in all channels and in 
all samples, but absent from the CD66abce/CD3 bivariate plot. Recently, Rahman et al. [25] reported generalized nonspecific metalconjugated antibody binding by eosinophils during the staining of fixed whole-blood cells, resembling the pattern observed in our fixed whole-blood samples, suggesting that these double-positive cells were eosinophils. We further excluded CD66abce/CD3 double-positive eosinophils from mass cytometry analysis.

One spanning-tree progression analysis of density-normalized events (SPADE) [26] was performed on both blood and bone marrow data sets using 33 clustering channels. Cell nodes generated by SPADE were manually annotated accordingly to the rules shown in online supplementary Table 4 . We performed a deeper analysis of these nodes using our recently released SPADEVizR R-package [27], dedicated to cytometry data analysis and focusing primarily on SPADE-derived data. The SPADE nodes were categorized by calculating the 1st and 99th percentiles of the expression distribution of the total cells for each marker and dividing this range into five expression categories (online suppl. Fig. 1). The mean of the median marker expression of cells contained in each node was then used to assign the expression of each marker to one of the five categories. Data for individuals with less than 50 events in a given node were excluded from the calculation to avoid biasing the phenotypical characterization of these small cell nodes. Hierarchical clustering was performed using the Euclidean metric and complete linkage method we recently published [27].

Flow Cytometry Cell Preparation and Phagocytosis Assay

Briefly, heparinized whole blood was processed within $1 \mathrm{~h}$ following blood draw to preserve neutrophils. For the phagocytosis assay, $100 \mu \mathrm{L}$ whole blood was incubated for $15 \mathrm{~min}$ on ice or in a $37^{\circ} \mathrm{C}$ water bath with Escherichia coli fluorescent bioparticules (pHrodo ${ }^{\mathrm{TM}}$ BioParticles, ThermoFisher) at a 20:1 ratio of particles to phagocytes. Phagocytosis was stopped by transferring the samples to ice and the addition of $20 \mathrm{ng}$ cytochalasin $\mathrm{D}$, inhibiting actin polymerization and further stopping phagocytosis of bioparticles. For phenotypic analysis and the phagocytosis assay, viability staining was first performed for $15 \mathrm{~min}$ at room temperature, according to the manufacturer's protocol (Live Dead, ThermoFisher). Then, antibody staining was performed with the following antibodies for $15 \mathrm{~min}$ at RT: CD64 BUV732 (10.1, BD Bioscience), CD11b V450 (ICRF144, BD Bioscience), CD3 BV605 (SP34.2, BD Bioscience), CD8a BV605 (RPAT8, BD Bioscience), CD20 BV605 (2H7, BD Bioscience), CD62L BV711 (SK11, BD Bioscience), CD32abc BV786 (FLI8.26, BD Bioscience), CD14 FITC (M5E2, BD Bioscience), CD16 PerCP-Cy55 (3G8, BD Bioscience), CDw125 PE (A14, BD Bioscience), CXCR4 PE-Dazzle 594 (12G5, Biolegend), CD89 PE-Cy7 (A59, Biolegend), HLADR AF700 (L234, Biolegend), and CD66abce APC-Vio770 (TET2, Myltenyi Biotec). Samples were then lysed and fixed using BD fluorescence-activated cell sorting (FACS) Lysing (BD Bioscience) for 15 min. After an additional wash with PBS, acquisition was performed on a BD FORTESSA flow cytometer (BD Bioscience) and analyzed

Fig. 2. Bone marrow and blood leukocyte characterization by mass cytometry. The SPADE tree shows the global analysis of leukocyte populations in blood and bone marrow from uninfected and chronically SIV-infected macaques. a The topology of the SPADE tree is shown with the cluster number associated with each node and color according to manually annotated leukocyte populations. using FlowJo software. The fluorescence of the bioparticles increases with acidification of the phagolysosome. Thus, we measured neutrophil phagocytosis by determining the percentage of pHrodo-positive PMNs. Then, we calculated the phagocytosis ratio to remove background fluorescence as follows:

$$
\frac{\% \text { pHrodo PMN at } 37^{\circ}-\% \text { pHrodo PMN at } 0{ }^{\circ} \mathrm{C}}{\% \text { pHrodo PMNsat } 0^{\circ} \mathrm{C}} \text {. }
$$

\section{Neutrophil Cell Sorting and Cytology}

For cell sorting by flow cytometry, whole blood or bone marrow from two uninfected animals were first $\mathrm{NH} 4 \mathrm{Cl}$ lysed, then $\mathrm{FcR}$ were blocked using cynomolgus macaque serum. Cells were counted and incubated $30 \mathrm{~min}$ with the following antibodies: CD11b (ICRF44), CD45 (D058-1283), CDw125 (A14), CD3 (REA994), CD20 (LT20), CD8a (BW135/80), CD14 (TUK4) CD32a (IV.3), and CD66 (TET2). Cell sorting was performed on FACSAria I flow cytometer (Becton Dickinson). Sorted populations were cytospined and then stained by May-Grünwald-Giemsa. Pictures were taken by a Nikon Eclipse 80 i with Dxm 1200C digital camera at $60 \times$ magnification. Cells were identified by morphological criteria by a cytologist. Myeloblasts, promyelocytes, and myelocytes were considered as pre-neutrophils, metamyelocytes, and band cells as immature neutrophils and segmented neutrophils as mature.

\section{Results}

\section{Identification of Leukocyte Changes during}

SIVmac251 Infection by Mass Cytometry

We first performed a pilot study in animals in the late chronic phase of SIV infection (18 months) to maximize the chance of unraveling major changes among cell subsets by multidimensional mass cytometry analysis. We used an unsupervised computational approach to objectively uncover cellular phenotypic heterogeneity from single-cell high-dimensional data (Fig. 1). SPADE analysis allows the organization of cells into a hierarchy of related phenotypes, forming cell clusters with close phenotypic profiles [26]. We created a 100-cluster common SPADE tree, which recapitulates the phenotypes of blood and bone marrow cell populations, to investigate the impact of SIVmac251 infection in the macaques. Clusters were grouped based on major cluster determinant markers (Fig. 2 and online suppl. Table 4), such as neutrophils, basophils, T lymphocytes, B lymphocytes, monocytes,
cDC, classical dendritic cells; pDC, plasmacytoid dendritic cells. b The tree was built using all samples and split according to the compartment and SIV status. Each dot represents a cluster with the size proportional to the cell abundance among all cells. SIV infection led to changes in the abundance of neutrophil clusters in both blood and bone marrow.

(For figure see next page.) 


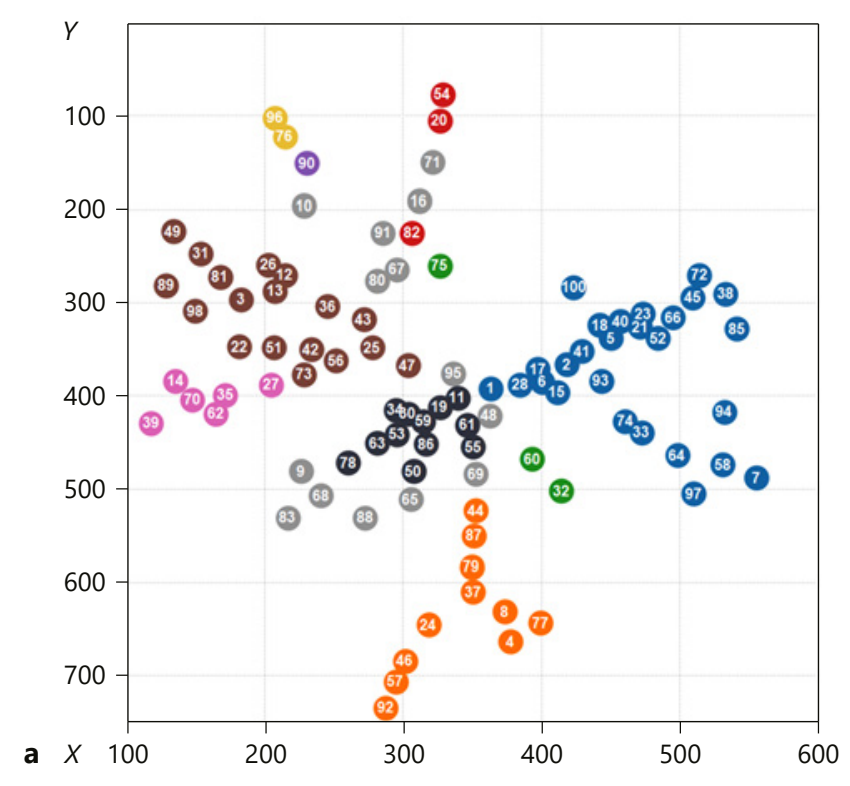

Cell population

T lymphocytes CD66- CD3+

B lymphocytes CD20+ HLADR+

NK cells CD3-CD8+

Neutrophils CD66+ CD3-

Basophils CD66- HLADR- CD123+

- Monocytes CD14+ HLADR+

CDC CD14- HLADR+ CD11C+ CD16+

pDC CD66- HLADR+ CD123+

- CD45 low

Undetermined

Percentage of total cells

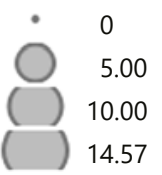

SIV+

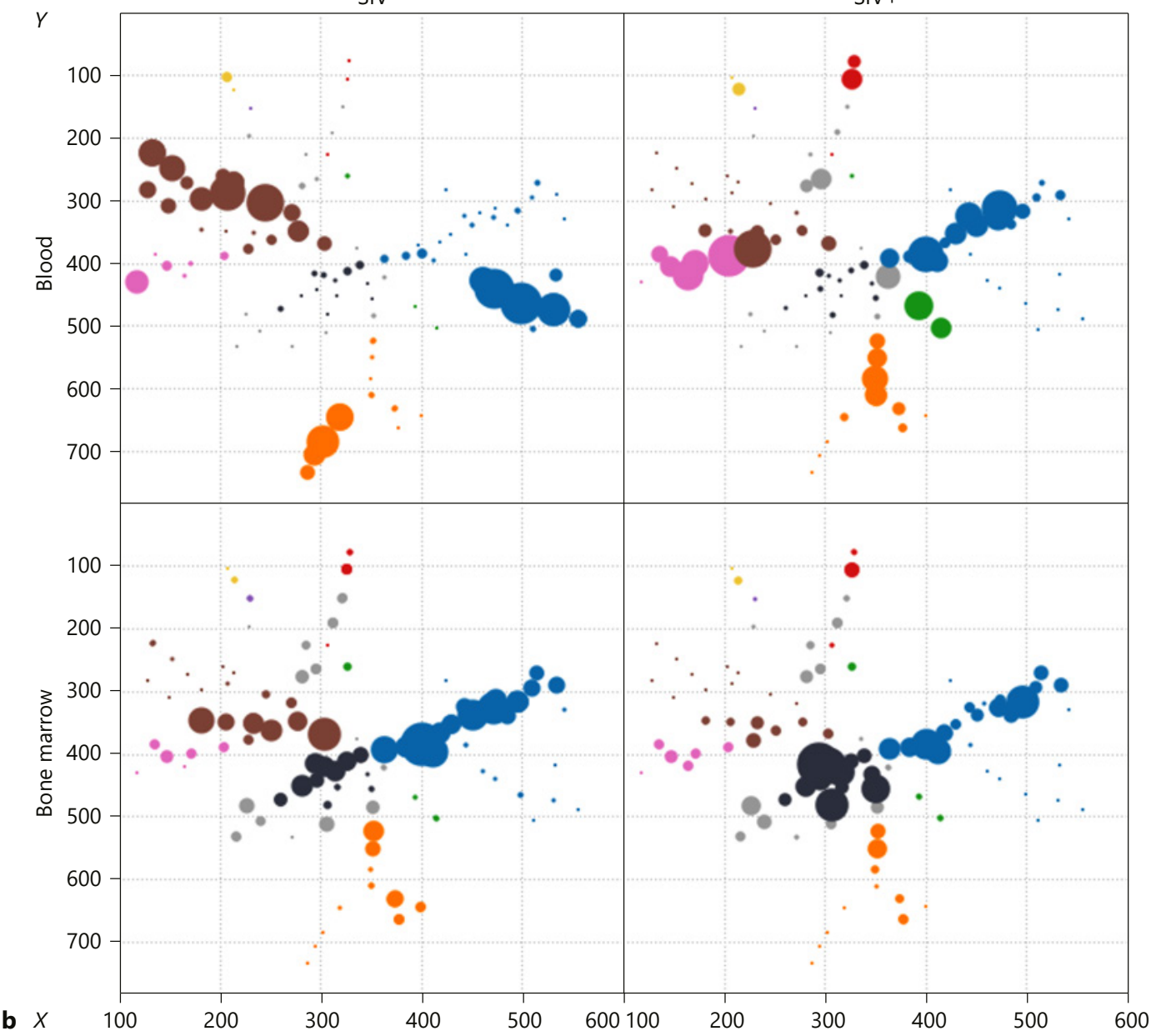


classical dendritic cells, and plasmacytoid dendritic cells. The study focused on myeloid cells. Thus, few markers for lymphoid cells were included, resulting in a limited number of T-, B-, and NK-cell clusters determined by the SPADE algorithm.

Despite the lack of lymphocyte markers, we observed already described CD4 T-cell, CD8 T-cell, and NK-cell phenotypes that characterize chronic SIV and HIV infection, thus validating the mass cytometry approach and analysis pipeline we used. Late chronic SIV infection affected peripheral T cells, decreasing the abundance of five clusters of CD4 T cells (\#3, 12, 13, 26, and 36) in SIV-infected individuals (Fig. 2), as expected. Clusters 3, 12, and 13 were characterized by CD $25^{\text {high }}$ CCR $7^{\text {high }}$ CD $29^{\text {mid/high }}$ cells, which disappeared from the blood (online suppl. Fig. 1). CD8 T-cell clusters (\#73, 42, and 22) that increased during late chronic SIV infection were CX3CR $1^{\text {high }}$, a marker previously correlated with cytotoxic function and effector differentiation [28]. NK-cell clusters enriched during late chronic SIV infection (\#14, 35, and 70) expressed granzyme and perforin, displaying a cytotoxic profile. Peripheral B-lymphocytes had a specific phenotypic pattern during SIV infection (Fig. 2). The bone marrow of infected monkeys showed a striking decrease in $\mathrm{T}$ cells, associated with an increase in CD45 cell subsets.

\section{Mass Cytometry Shows Neutrophils to Be Diverse and Immature during Late Chronic SIV Infection}

We focused our analysis on PMNs, as mass cytometry associated with SPADE analysis revealed strong heterogeneity of PMN populations in blood and bone marrow. Indeed, 28 of 100 clusters were classified as PMNs, based on the expression of CD66 and the lack of other lineage markers.

In the uninfected control group, PMN clusters were separated into two distinct branches, depending on blood or bone marrow origin (Fig. 2). Bone marrow and blood PMNs had clearly distinct phenotypes, as expected. Peripheral PMNs appeared to be highly affected by infection, showing a clear enrichment of bone-marrow pheno-

Fig. 3. Determination of neutrophil maturation stage during SIV infection. a Neutrophil maturation stage and phenotype according to compartment. The relative expression of CD11b, CD32, CD45, CD66, and CXCR4 allow us to split neutrophils into three populations according to maturation: pre-neutrophils, immature neutrophils, and mature neutrophils. b FACS gating strategy and representative May-Grünwald-Giemsa-stained cytospin of sorted CD11b-CD32a- pre-neutrophils, CD11b+CD32a ${ }^{\text {mid }}$ immature and $\mathrm{CD} 11 \mathrm{~b}+\mathrm{CD} 32 \mathrm{a}^{\text {high }}$ mature neutrophils. c SPADE tree from

Immature Neutrophils in SIV Infection type. Neutrophil granulopoiesis can be divided into three different maturation stages based on proliferation capacities, phenotype, and cell morphology [29]. Pre-neutrophils are PMN precursors with proliferative capacities, which further differentiate into immature and mature neutrophils. We were able to associate cluster phenotypes by neutrophil maturation stage in bone marrow from the uninfected control group based on CD66, CD45, CXCR4, CD32, and CD11b expression profiles (Fig. 3a) [30, 31]. Using FACS, in combination with cytospin, we were able to confirm cell morphology associated with maturation phenotypes identified by mass cytometry in blood and bone marrow (Fig. 3b). Pre-neutrophil phenotype was associated with myeloblasts, promyelocytes, and myelocytes in May-Grünwald-Giemsa-stained cytology. Immature phenotype was mainly associated with metamyelocyte/band cell cytology, and the mature fraction contained segmented neutrophils. Frequency of pre-neutrophils, immature and mature neutrophils obtained in FACS analysis were consistent with mass cytometry data. Bone marrow had highly abundant pre-neutrophils and immature neutrophils (Fig. 3b, c) [29, 32], as expected. Mass cytometry analysis was also able to detect a small number of pre-neutrophils and immature neutrophils in the blood of uninfected macaques, with the dominant population being mature neutrophils, as expected. Late chronic SIV infection led to a redistribution of neutrophils in the blood and bone marrow. Mature neutrophils were no longer present in the circulation and were replaced by immature neutrophils (Fig. 3c). This global change in neutrophil maturation was characterized by low expression of CD11b, CD32, CD45, and CD66 (Fig. 3a). In addition, bone marrow pre-neutrophils were more abundant in chronically SIV-infected animals.

\section{Neutrophil Priming Occurs during Early Chronic SIV}

Infection, Shown by the Impairment of Phagocytosis

We then characterized the redistribution of neutrophils at earlier stages of infection and the impact of cART by analyzing peripheral blood from $15 \mathrm{SIV}$-infected and 9 un-

Figure 1, focusing on neutrophil clusters with the associated cluster number and colored by maturation stage. Abundance of each neutrophil maturation stage among leukocytes from the uninfected control group and SIV-infected animals. SIV infection induced a decrease in the abundance of mature neutrophils in blood and an enrichment of immature neutrophils in blood. Clusters with less than 150 cells were excluded, and cluster annotation was performed according to online supplementary Table 4.

(For figure see next page.)

J Innate Immun 2020;12:170-181 

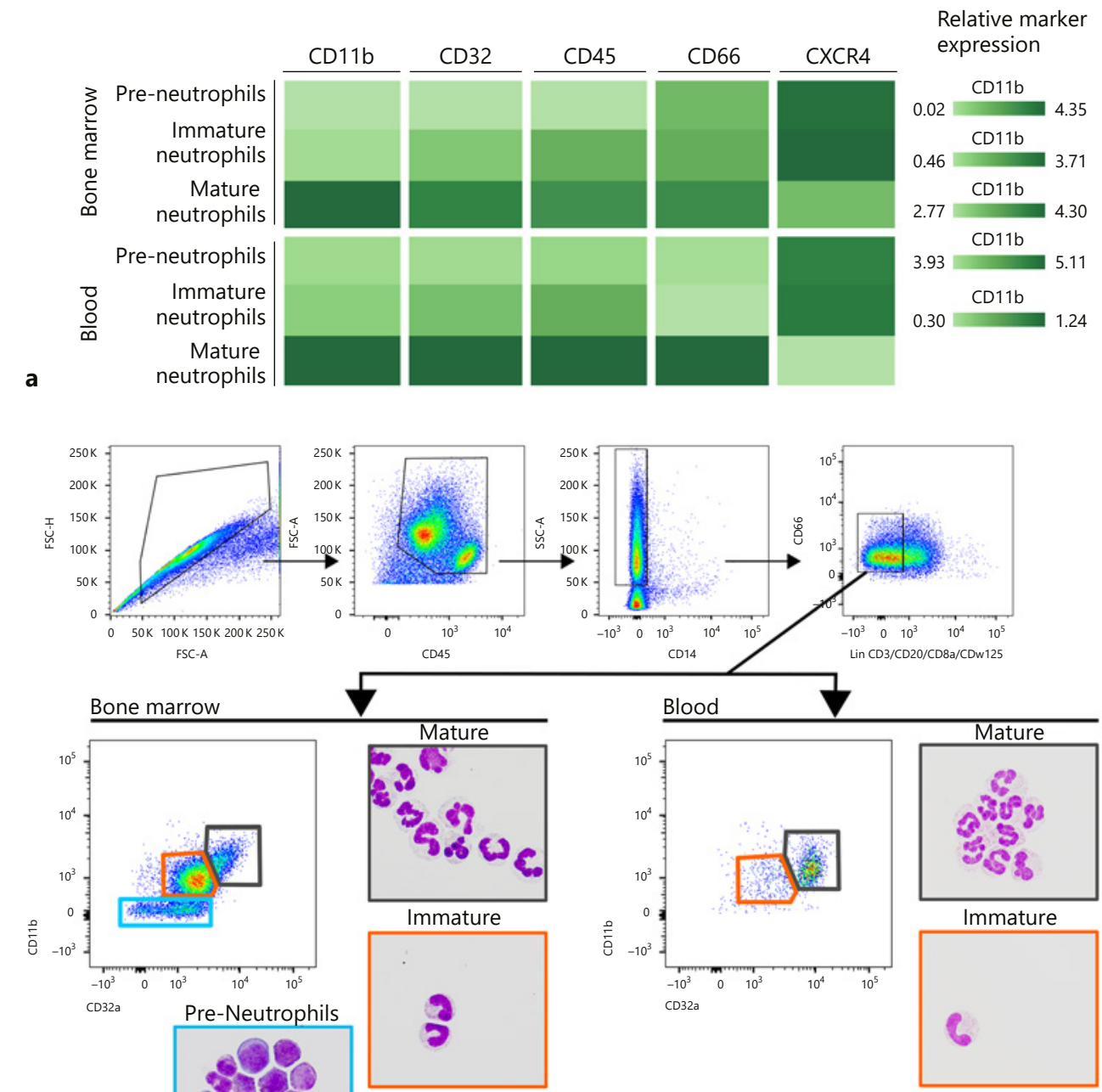

b
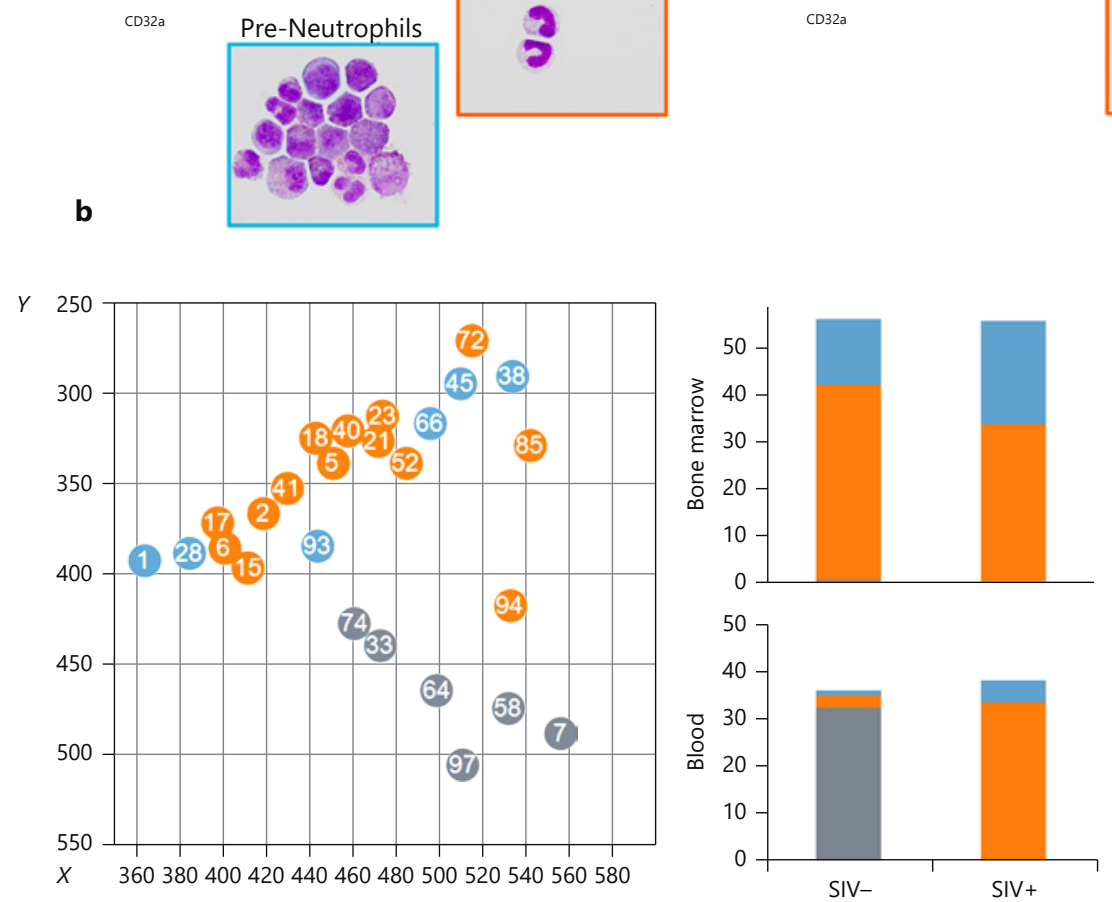

Neutrophil maturation - Pre-neutrophils

Immature neutrophils

Mature neutrophils

C $\quad X \quad 360380400420440460480500520540560580$ 


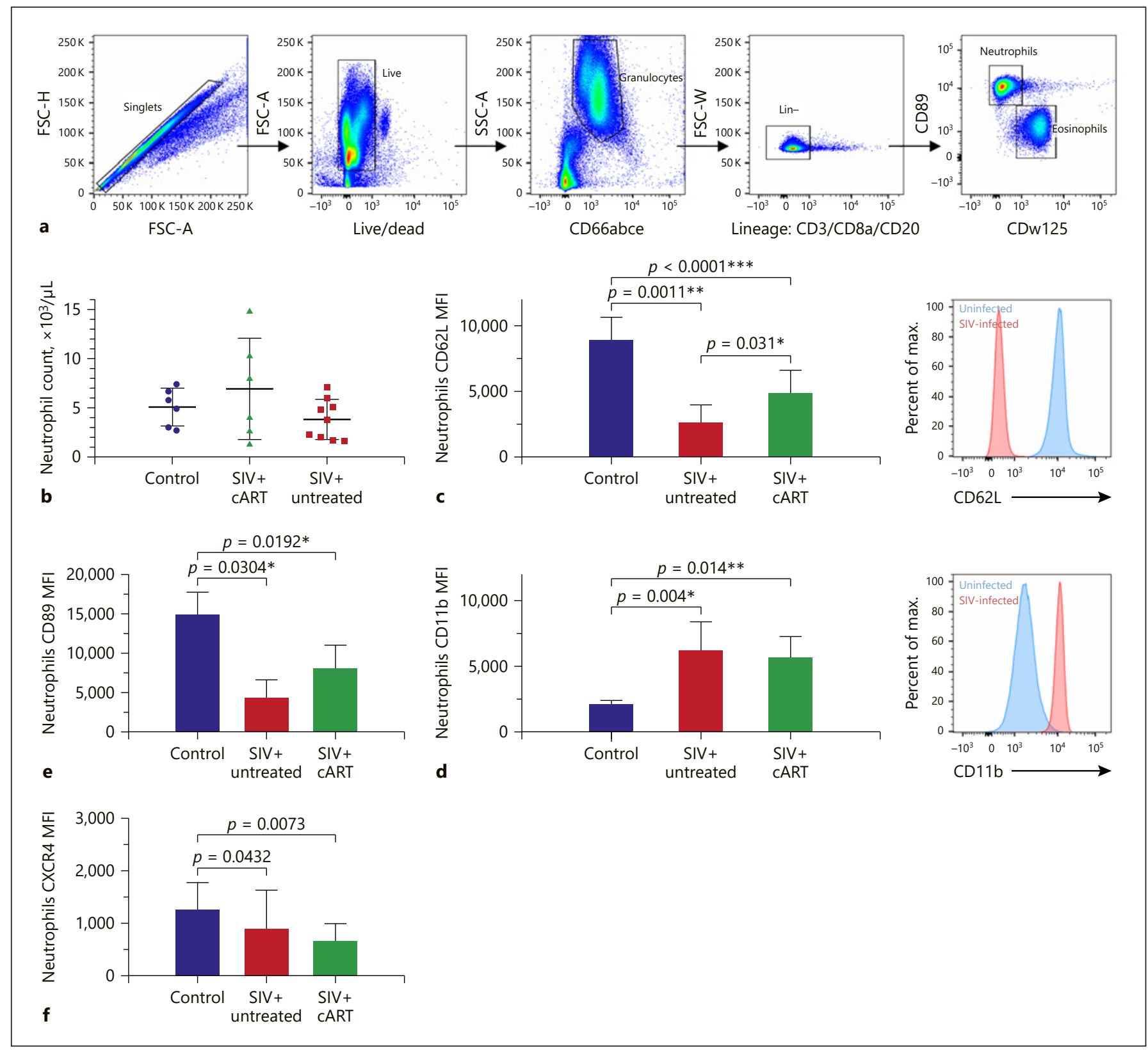

Fig. 4. SIV infection induces neutrophil priming during the early chronic phase, which is partially restored by treatment. a Gating strategy used in cynomolgus macaques to isolate neutrophils from eosinophils by flow cytometry. b Neutrophil blood count in three groups. c, d CD11b and CD62L marker expression in blood neu-

infected macaques by conventional flow cytometry. They included 12 macaques 120 days after infection, with 6 animals under cART from day 28 of infection; the remaining 3 macaques were at 288 days of infection, without cART. We identified neutrophils as CD66abce positive, lineage negative (CD3, CD20, and CD8a), CD89 positive, and trophils. SIV infection-induced neutrophil priming during the early chronic phase was partially restored by cART. e, f CXCR 4 and CD89 MFI of blood neutrophils from uninfected control, untreated SIV-infected, and treated SIV-infected macaques. KruskalWallis test and Dunn's multiple comparison test.

CDw125 negative, due to the lack of a CD66b antibody that cross-reacts with cynomolgus macaque cells (Fig. 4a).

There was no difference in the neutrophil count from whole blood between control, SIV+ cART and SIV+ untreated groups (Fig. 4b). Neutrophil CD66 and CD32 expression was similar between groups, showing no differ- 


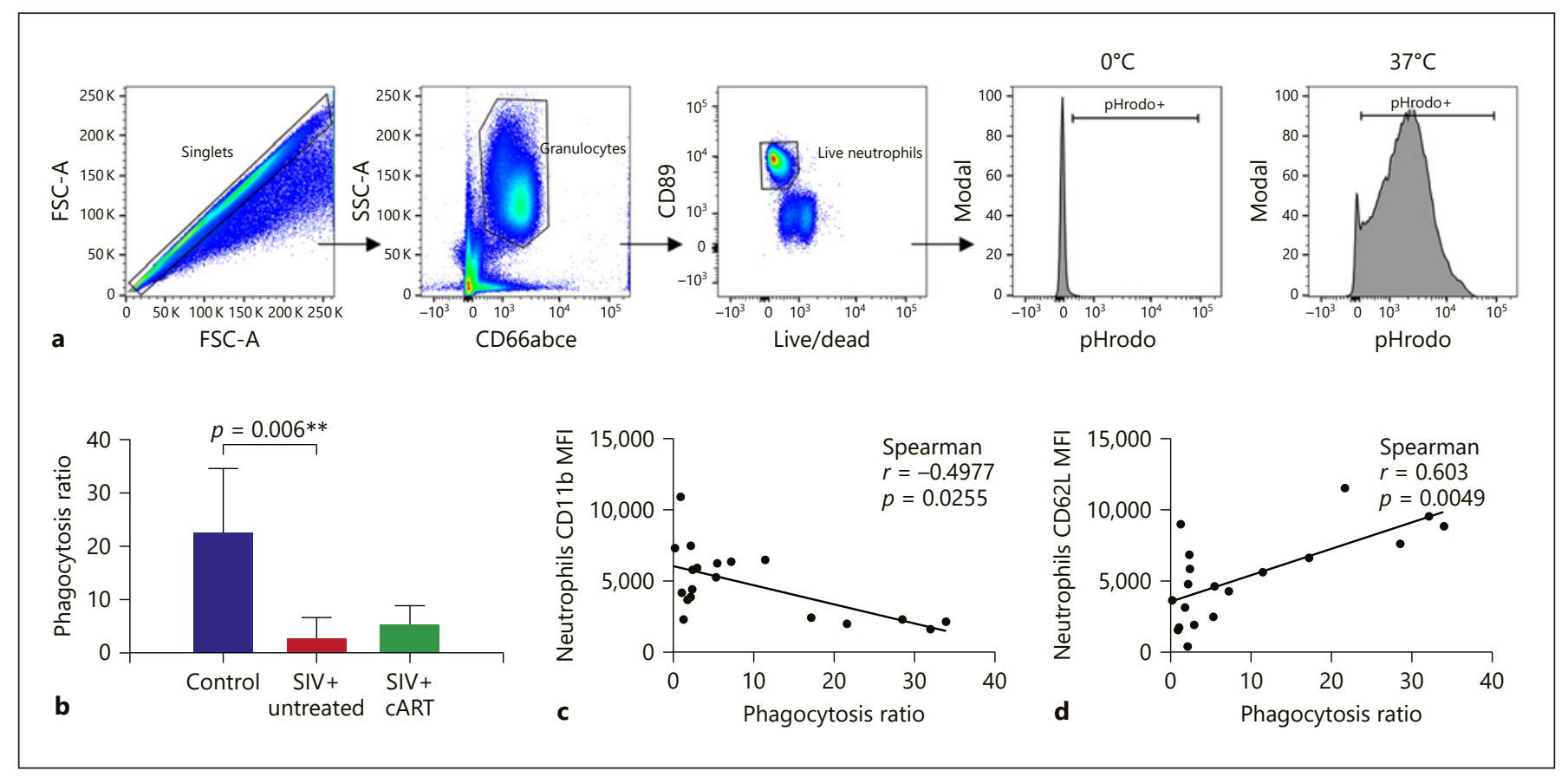

Fig. 5. Neutrophil phagocytosis during early chronic infection. a Gating strategy used to analyze neutrophil phagocytosis in cynomolgus macaques and $E$. coli bioparticle fluorescence measurement. b Phagocytosis ratio of blood neutrophils in the three groups. Neutrophil phagocytosis decreased during early chronic

ence in terms of maturation during the early stages of infection (online suppl. Fig. 2). We analyzed CD62L shedding and the increase in CD11b expression, which have been linked to priming [33], between PMN of treatment groups in order to identify changes in their activation state associated with SIV infection. Neutrophil CD11b expression was significantly higher for both SIV-infected groups (treated, $p=0.014$, and untreated, $p=0.004$, Fig. 4d) than that for uninfected controls. In parallel, CD62L expression was lower for all infected animals, suggesting an increased PMN priming during the early stages of SIV infection (treated: $p<0.0001$, untreated: $p=0.0011$, Fig. 4c) [34]. Differential expression of CD62L and CD11b was partially reduced by cART, as shown by higher CD62L expression, but CD11b expression was unaffected. Neutrophils from early treated and untreated SIV-infected macaques showed a significantly lower expression of CXCR4 than those of uninfected macaques $(p=0.0073$, Fig. 4f). Moreover, SIV infection induced an increase in CD89 (Fca-RI) expression, which was not corrected by cART (Fig. 4e). Thus, neutrophils displayed altered expression of surface molecules, with modulation of the chemokine receptor CXCR4 and CD89 Fc receptor levels.
SIV infection, and treatment initiation during primary infection could not restore phagocytosis. c Correlation between the MFI of CD11b and CD62L of neutrophils with phagocytosis. Spearman test and Kruskal-Wallis associated with Dunn's multiple comparison test.

We characterized phagocytosis of whole blood neutrophils to determine whether priming modulates neutrophil function. We used bacterial particles coated with a $\mathrm{pH}$-dependent fluorescent dye, allowing us to measure phagocytosis by following acidification of the phagosome (Fig. 5a). The phagocytic capacity of neutrophils of untreated SIVinfected animals was significantly less than that of the uninfected control group (Fig. 5b). This decrease in phagocytosis correlated inversely with CD11b expression (Fig. 5c) and positively with CD62L expression (Fig. 5d) but not with CXCR4 or CD89 expression, a finding consistent with the idea that exposure of neutrophils to SIV causes functional impairment. Early cART initiation failed to restore $\mathrm{PMN}$ phagocytosis in the treated group.

\section{Discussion}

We describe, for the first time, the diversity of the leukocyte population in the blood and bone marrow of SIVinfected macaques using multiparametric mass cytometry. Such analysis highlights numerous modifications of the repartition of leukocyte subsets. Neutrophils were par- 
ticularly heterogeneous, with distinct complex phenotypes in blood and bone marrow. We used myeloid markers and published phenotypes [35-38] to differentiate preneutrophils, with proliferative capacities, from immature and mature neutrophils in a cynomolgus macaque model of HIV infection. During late chronic SIV infection, immature bone marrow neutrophils were found in the blood, showing mobilization of the medullary neutrophil pool, as described in emergency granulopoiesis and sepsis [37]. Immature neutrophils were the most abundant circulating granulocyte populations found in SIV-infected animals. Moreover, mature neutrophils almost completely disappeared from the blood and bone marrow, probably undergoing apoptosis or tissue mobilization [39]. It will be necessary for future studies to determine whether functions differ between mature and immature PMN subpopulations, and if so, whether they are functionally different populations during SIV infection.

Mass cytometry showed elevated levels of the chemokine receptor CXCR4 in bone marrow neutrophils and subsets of peripheral neutrophils from chronically SIVinfected macaques. At steady state, CXCR4 maintains neutrophils in the bone marrow by interacting with CXCL12 expressed on endothelial cells [30]. CXCL12 is downregulated when inflammation occurs or upon G-CSF signaling in endothelial cells, resulting in neutrophil mobilization. CXCR4 expression decreases in young circulating neutrophils [40] and aged neutrophils re-express CXCR4, allowing them to home to specific sites and be cleared by macrophages in the spleen and bone marrow [41]. It is difficult to determine whether neutrophils are immature or aged based on CXCR4 expression alone. However, the association of strong CXCR4 expression with $\mathrm{CD} 66, \mathrm{CD} 32$, and $\mathrm{CD} 45$ suggests that the circulating neutrophils were immature, just having been mobilized from the bone marrow. Mature neutrophils were no longer present in the blood of chronically infected macaques, indicating apoptosis of aged neutrophils or mobilization to tissues where viral replication sustains inflammation.

Reducing the number of markers in flow cytometry abolished the ability to differentiate immature from mature neutrophils in blood as the neutrophil population was homogeneous in terms of CD66 and CD32 expression. Other markers are necessary to better delineate neutrophil maturation, such as CD49d, CD101, and CD10, recently described to distinguish between pre-neutrophils, immature neutrophils, and mature neutrophils in human blood and bone marrow [32].

The combination of mass cytometry and flow cytometry results suggests that CXCR4 expression on neutro-

Immature Neutrophils in SIV Infection phils evolves, depending on the stage of SIV infection and the neutrophil subset: CXCR4 downregulation during early phases, probably due to the presence of young primed neutrophils, followed by an increase in CXCR4 expression during the late stages of infection to normal levels or higher, due to the massive egress of immature neutrophils from the bone marrow and the presence of rare aged mature neutrophils.

Neutrophils observed during the early chronic phase of SIV infection had changes in surface expression of CD62L and CD11b, which have been previously associated with priming [33]. There is a continuum of PMN activation from priming to full activation depending on the stimulus intensity [42]. Priming is a complex mechanism in which the environment induces changes in neutrophil responsiveness to stimuli. Priming relies on mobilization of secretory vesicles and gelatinase granules, whereas fully activated neutrophils are characterized by NAPDH oxidase activation along with azurophilic granule release [43]. In this study, the analysis of CD62L and $\mathrm{CD} 11 \mathrm{~b}$ alone did not allow conclusions to be drawn about the extent of PMN activation following exposure to SIV.

In the early chronic phase, PMN phagocytic capacity was also impaired. The oxidative burst in neutrophils and phagocytosis correlated with viral load, CD4 T-cell count, and treatment success, as reported in a recent study [12]. In the same study, the phagocytic activity in HIV-infected patients was significantly less than that of healthy individuals, but without a beneficial effect of cART. However, patients from this cohort had advanced chronic HIV infection, and treatment was initiated long after transmission. We demonstrated that neutrophil phagocytosis was impaired from early chronic SIV infection using E. coli bioparticles. Treatment initiated 1 month after inoculation did not restore neutrophil phagocytosis in SIV-infected animals. Early cART partially reduced the impact on CD62L and CD11b expression, but neutrophil phagocytosis was still impaired, probably because of chronic immune activation. Since the phagocytosis assay is based on acidification, it is also possible that neutrophils lost their capacities to acidify their vacuoles during SIV infection, making them less potent pathogen killers. Neutrophil priming must be explored in further detail, to understand the inflammatory environment associated with SIV infection which shapes their functionality and in turn fuels chronic immune activation.

The use of mass cytometry allowed us to identify numerous neutrophil subsets and show the presence of immature populations during the chronic phases of SIV infection. The monitoring of immature circulating neutrophils is performed for the diagnosis and prognosis of patients with sys- 
temic inflammatory response syndrome [44]. Patients with high levels of myelocytes and metamyelocytes (called myelocytosis) had a poor outcome, regardless of whether the origin was infectious or noninfectious. Whether myelocytosis is associated with HIV progression or treatment escape could be determined, as a blood smear could be a simple and valuable bedside prognostic tool. Moreover, we demonstrated that CART initiated during primary infection could not reduce neutrophil priming and function. Whether neutrophils play a role in the establishment and maintenance of chronic immune activation should be explored, as neutrophils represent $40-60 \%$ of circulating leukocytes.

\section{Acknowledgments}

This work was supported by the "Programme Investissements d'Avenir" (PIA), managed by the ANR under reference ANR11-INBS-0008, funding the Infectious Disease Models and Innovative Therapies (IDMIT, Fontenay-aux-Roses, France) infrastructure, and ANR-10-EQPX-02-01, funding the FlowCyTech facility (IDMIT, Fontenay-aux-Roses, France). We thank Gilead and ViiV for providing the antiretroviral drugs. Animal studies were supported by the ANRS and MSD Avenir, as part of the pVISCONTI research program, and the SIVART ANRS-IDMIT CO1 research program. We thank all members of the FlowCyTech, Animal Science and Welfare, and Infectiology Immunology Laboratory core facilities of the IDMIT infrastructure for their excellent expertise and outstanding contribution.

\section{Disclosure Statement}

The authors have no conflicts of interest to declare.

\section{Author Contributions}

R.L.G., O.L., and J.L. designed and supervised the study. J.L. performed experiments and discussed the data. R.L.G., O.L., D.D., and A.C. discussed the data and read the paper. J.L. wrote the paper.

\section{References}

1 Freiberg MS, Chang CC, Kuller LH, Skanderson M, Lowy E, Kraemer KL, et al. HIV infection and the risk of acute myocardial infarction. JAMA Intern Med. 2013 Apr;173(8): 614-22.

2 Nasi M, Pinti M, De Biasi S, Gibellini L, Ferraro D, Mussini C, et al. Aging with HIV infection: a journey to the center of inflammAIDS, immunosenescence and neuroHIV. Immunol Lett. 2014 Nov;162(1 1 Pt B):32933.

3 Deeks SG, Tracy R, Douek DC. Systemic effects of inflammation on health during chronic HIV infection. Immunity. 2013 Oct;39(4): 633-45.

4 Burdo TH, Lo J, Abbara S, Wei J, DeLelys ME, Preffer F, et al. Soluble CD163, a novel marker of activated macrophages, is elevated and associated with noncalcified coronary plaque in HIV-infected patients. J Infect Dis. 2011 Oct;204(8):1227-36.

5 Kelesidis T, Kendall MA, Yang OO, Hodis HN, Currier JS. Biomarkers of microbial translocation and macrophage activation: association with progression of subclinical atherosclerosis in HIV-1 infection. J Infect Dis. 2012 Nov;206(10):1558-67.

6 So-Armah KA, Tate JP, Chang CH, Butt AA, Gerschenson M, Gibert CL, et al.; VACS Project Team. Do biomarkers of inflammation, monocyte activation, and altered coagulation explain excess mortality between hiv infected and uninfected people? J Acquir Immune Defic Syndr. 2016 Jun;72(2):206-13.
7 Brenchley JM, et al. Microbial translocation is a cause of systemic immune activation in chronic HIV infection. Nat Med. 2006;12:1365-71.

8 Wright HL, Moots RJ, Edwards SW. The multifactorial role of neutrophils in rheumatoid arthritis. Nat Rev Rheumatol. 2014 Oct; 10(10):593-601.

9 Campillo-Gimenez L, Casulli S, Dudoit Y, Seang S, Carcelain G, Lambert-Niclot S, et al. Neutrophils in antiretroviral therapy-controlled HIV demonstrate hyperactivation associated with a specific IL-17/IL-22 environment. J Allergy Clin Immunol. 2014 Nov;134(5):1142-52.e5.

10 Elbim C, Monceaux V, François S, Hurtrel B, Gougerot-Pocidalo MA, Estaquier J. Increased neutrophil apoptosis in chronically SIV-infected macaques. Retrovirology. 2009 Mar;6(1):29.

11 Pos O, Stevenhagen A, Meenhorst PL, Kroon FP, Van Furth R. Impaired phagocytosis of Staphylococcus aureus by granulocytes and monocytes of AIDS patients. Clin Exp Immunol. 1992 Apr;88(1):23-8.

12 Michailidis C, Giannopoulos G, Vigklis V, Armenis K, Tsakris A, Gargalianos P. Impaired phagocytosis among patients infected by the human immunodeficiency virus: implication for a role of highly active anti-retroviral therapy. Clin Exp Immunol. 2012 Mar;167(3):499-504.

13 Bowers NL, Helton ES, Huijbregts RP, Goepfert PA, Heath SL, Hel Z. Immune suppression by neutrophils in HIV-1 infection: role of PD-L1/PD-1 pathway. PLoS Pathog. 2014 Mar;10(3):e1003993.
14 Matusali G, Dereuddre-Bosquet N, Le Tortorec A, Moreau M, Satie AP, Mahé D, et al. Detection of Simian Immunodeficiency Virus in Semen, Urethra, and Male Reproductive Organs during Efficient Highly Active Antiretroviral Therapy. J Virol. 2015 Jun;89(11): 5772-87.

15 Mannioui A, Bourry O, Sellier P, Delache B, Brochard P, Andrieu T, et al. Dynamics of viral replication in blood and lymphoid tissues during SIVmac251 infection of macaques. Retrovirology. 2009 Nov;6(1):106.

16 Bruel T, Dupuy S, Démoulins T, RogezKreuz C, Dutrieux J, Corneau A, et al. Plasmacytoid dendritic cell dynamics tune interferon-alfa production in SIV-infected cynomolgus macaques. PLoS Pathog. 2014 Jan; 10(1):e1003915.

17 Bruel T, Hamimi C, Dereuddre-Bosquet N, Cosma A, Shin SY, Corneau A, et al. Longterm control of simian immunodeficiency virus (SIV) in cynomolgus macaques not associated with efficient SIV-specific CD8+ T-cell responses. J Virol. 2015 Apr;89(7):3542-56.

18 Policicchio BB, Pandrea I, Apetrei C. Animal models for HIV cure research. Front Immunol. 2016 Jan;7:12.

19 Elhmouzi-Younes J, Palgen JL, Tchitchek N, Delandre S, Namet I, Bodinham CL, et al. In depth comparative phenotyping of blood innate myeloid leukocytes from healthy humans and macaques using mass cytometry. Cytometry A. 2017 Oct;91(10): 969-82. 
20 Bandura DR, Baranov VI, Ornatsky OI, Antonov A, Kinach R, Lou X, et al. Mass cytometry: technique for real time single cell multitarget immunoassay based on inductively coupled plasma time-of-flight mass spectrometry. Anal Chem. 2009 Aug;81(16): 6813-22.

21 Pereyra F, Jia X, McLaren PJ, Telenti A, de Bakker PI, Walker BD, et al.; International HIV Controllers Study. The major genetic determinants of HIV-1 control affect HLA class I peptide presentation. Science. 2010 Dec; 330(6010):1551-7.

22 Karlsson I, Malleret B, Brochard P, Delache B, Calvo J, Le Grand R, et al. Dynamics of T-cell responses and memory $\mathrm{T}$ cells during primary simian immunodeficiency virus infection in cynomolgus macaques. J Virol. 2007 Dec; 81(24):13456-68.

23 Del Prete GQ, Smedley J, Macallister R, Jones GS, Li B, Hattersley J, et al. Short Communication: Comparative Evaluation of Coformulated Injectable Combination Antiretroviral Therapy Regimens in Simian Immunodeficiency Virus-Infected Rhesus Macaques. AIDS Res Hum Retroviruses. 2016 Feb;32(2): 163-8.

24 Finck, R. et al. Normalization of mass cytometry data with bead standards. Cytometry A. 2013;83(5):483-94.

25 Rahman AH, Tordesillas L, Berin MC. Heparin reduces nonspecific eosinophil staining artifacts in mass cytometry experiments. Cytometry A 2016;89(6):1-7.

26 Qiu P, Simonds EF, Bendall SC, Gibbs KD Jr, Bruggner RV, Linderman MD, et al. Extracting a cellular hierarchy from high-dimensional cytometry data with SPADE. Nat Biotechnol. 2011 Oct;29(10):886-91.
27 Gautreau G, Pejoski D, Le Grand R, Cosma A, Beignon AS, Tchitchek N. SPADEVizR: an R package for visualization, analysis and integration of SPADE results. Bioinformatics. 2017 Mar;33(5):779-81.

28 Gerlach C, Moseman EA, Loughhead SM, Alvarez D, Zwijnenburg AJ, Waanders L, et al. The Chemokine Receptor CX3CR1 Defines Three Antigen-Experienced CD8 T Cell Subsets with Distinct Roles in Immune Surveillance and Homeostasis. Immunity. 2016 Dec;45(6):1270-84.

29 Bolliger AP, Fontaine M. Cytological examination and cellular composition of bone marrow in healthy, adult, cynomolgus monkeys (Macaca fascicularis). Comp Haematol Int. 1998;8(4):183-90.

30 Borregaard N. Neutrophils, from marrow to microbes. Immunity. 2010 Nov;33(5):657-70.

31 Elghetany MT. Surface antigen changes during normal neutrophilic development: a critical review. Blood Cells Mol Dis. 2002 MarApr;28(2):260-74.

32 Evrard M, Kwok IW, Chong SZ, Teng KW, Becht E, Chen J, et al. Developmental analysis of bone marrow neutrophils reveals populations specialized in expansion, trafficking, and effector functions. Immunity. 2018 Feb; 48(2):364-79.e8

33 Condliffe AM, Chilvers ER, Haslett C, Dransfield I. Priming differentially regulates neutrophil adhesion molecule expression/function. Immunology. 1996 Sep;89(1):105-11.

34 Ivetic A. A head-to-tail view of L-selectin and its impact on neutrophil behaviour. Cell Tissue Res. 2018 Mar;371(3):437-53.

35 Blazkova J, Gupta S, Liu Y, Gaudilliere B, Ganio EA, Bolen CR, et al. Multicenter Systems Analysis of Human Blood Reveals Immature Neutrophils in Males and During Pregnancy. J Immunol. 2017 Mar;198(6):2479-88.
36 Otten MA, Rudolph E, Dechant M, Tuk CW, Reijmers RM, Beelen RH, et al. Immature neutrophils mediate tumor cell killing via IgA but not IgG Fc receptors. J Immunol. 2005 May;174(9):5472-80.

37 Drifte G, Dunn-Siegrist I, Tissières P, Pugin J. Innate immune functions of immature neutrophils in patients with sepsis and severe systemic inflammatory response syndrome. Crit Care Med. 2013 Mar;41(3):820-32.

38 Marini O, et al. Mature CD10+ and immature CD10 - neutrophils present in G-CSF-treated donors display opposite effects on T cells. Blood. 2017;129:1343-57.

39 Greenlee-Wacker MC. Clearance of apoptotic neutrophils and resolution of inflammation. Immunol Rev. 2016 Sep;273(1): 357-70.

40 Martin C, Burdon PC, Bridger G, GutierrezRamos JC, Williams TJ, Rankin SM. Chemokines acting via CXCR2 and CXCR4 control the release of neutrophils from the bone marrow and their return following senescence. Immunity. 2003 Oct;19(4):583-93.

41 Rankin SM. The bone marrow: a site of neutrophil clearance. J Leukoc Biol. 2010 Aug; 88(2):241-51.

42 Sengeløv H, Kjeldsen L, Borregaard N. Control of exocytosis in early neutrophil activation. J Immunol. $1993 \mathrm{Feb}$;150(4):1535-43.

43 Miralda I, Uriarte SM, Mcleish KR. Multiple phenotypic changes define neutrophil priming. Front Cell Infect Microbiol. 2017;7: 217.

44 Mare TA, Treacher DF, Shankar-Hari M, Beale R, Lewis SM, Chambers DJ, et al. The diagnostic and prognostic significance of monitoring blood levels of immature neutrophils in patients with systemic inflammation. Crit Care. 2015 Feb;19(1):57. 